\title{
EFEITOS DE TRÊS REPETIÇÕES DE IO SEGUNDOS DE ESTÍMULO DO MÉTODO ESTÁTICO PARA O DESENVOLVIMENTO DA FLEXIBILIDADE DE HOMENS ADULTOS JOVENS
}

\author{
MS. LUCIANE VOIGT \\ Mestre em Ciência da Motricidade Humana pela UCB/RJ e \\ Pesquisadora do Laboratório de Biociências da Motricidade \\ Humana (LABIMH/UNIRIO) (Rio de Janeiro - Brasil) \\ e-mail: luvoigt@oi.com.br
}

DR. RODRIGO GOMES DE SOUZA VALE

Doutor em Ciências da Saúde pela UFRN, Professor do Curso de Educação Física da Universidade Estácio de Sá (Cabo Frio-RJ-Brasil) e Pesquisador do Laboratório de Biociências da Motricidade Humana (LABIMH/UNIRIO) (Rio de Janeiro - Brasil) e-mail: rodrigo.vale@unirio.br

MS. DENNIS WILLIAM ABDALA

Mestre em Educação Física pela UNIMEP e Professor do Centro Universitário da Fundação Educacional Guaxupé (UNIFEG) (Minas Gerais - Brasil) e-mail: dennismestrado@yahoo.com.br

\section{MS. WAGNER ZEFERINO DE FREITAS}

Mestre em Ciência da Motricidade Humana pela UCB/RJ, Professor da Escola Agrotécnica Federal de Muzambinho (Minas Gerais - Brasil) e Pesquisador do Laboratório de Biociências da Motricidade Humana (LABIMH/UNIRIO) (Rio de Janeiro - Brasil) e-mail: wagnerzf@yahoo.com.br

\section{DR. JEFFERSON DA SILVA NOVAES}

Doutor em Educação Física pela UGF/RJ e Professor Associado da Escola de Educação Física e Desportos da Universidade Federal do Rio de Janeiro (EEFD/UFRJ) (Rio de Janeiro - Brasil) e-mail: jsnovaes@terra.com.br

\section{DR. ESTÉLIO HENRIQUE MARTIN DANTAS}

Doutor Livre Docente em Educação Física pela UERJ e Pesquisador do Laboratório de Biociências da Motricidade Humana (LABIMH/UNIRIO) (Rio de Janeiro - Brasil) e-mail: estelio.dantas@unirio.br 


\section{RESUMO}

Objetivo do estudo foi investigar os efeitos de três repetições de 10 segundos de permanência do método estático sobre os ganhos de amplitude de movimento em adultos jovens não atletas. Os 59 sujeitos foram divididos em um grupo experimental ( $G E ; n=4$ I), que foi submetido a exercícios de alongamento estático por 16 semanas, com aplicação de três repetições de 10 segundos de tempo de permanência na posição de cada movimento e um grupo controle (GC; $n=18$ ), que não foi submetido a nenhum treino. Foram avaliados os movimentos de abdução do ombro (AO) e flexão de quadril (FQ) através de goniometria. A ANOVA de medidas repetidas revelou aumentos significativos no GE do pré para o pós-teste no movimento $\mathrm{AO}(\%=8,9 ; p=0,00)$ e $\mathrm{FQ}(\%=18,7 ; p=0,00)$. No pós-teste não houve diferenças intergrupos. Conclui-se que o método proposto foi suficiente para produzir aumento significativo nos níveis de flexibilidade nos movimentos analisados.

PALAVRAS-CHAVE: Flexibilidade; alongamento; amplitude de movimento.

\section{INTRODUÇÃO}

A amplitude de movimento (ADM) articular pode ser considerada como um dos fatores determinantes para a eficácia na execução dos diferentes movimentos envolvidos na realização das atividades da vida diária (GONÇALVES; GURJÃO; GOBBI, 2007). Os exercícios utilizados para desenvolver a flexibilidade causam um aumento no comprimento da unidade músculo-tendão e as propriedades viscosas dos tecidos fazem com que este aumento não seja imediatamente reversível (VIVEIROS et al., 2004). Nesse sentido, recomenda-se que o treinamento desta qualidade física deva ser frequente para que a ADM se mantenha conservada por mais tempo (LIMA et al., 2006).

Dessa forma, um programa de treinamento de flexibilidade pode ser feito com exercícios de alongamento em níveis submáximos ou máximos de ADM, sendo este último dividido em métodos estático, dinâmico e facilitação neuromuscular proprioceptiva (FNP) (GUTIÉRREZ NIETO et al., 2003; HAFF, 2006).

O método estático parece ser o método mais utilizado pela sua facilidade de aplicação, aprendizado e segurança (OSTERNING; ROBERTSON; TROXEL, 1990). Pode ser aplicado em ambiente esportivo e terapêutico para proporcionar melhora da aptidão corporal e postura, alívio de cãibras musculares, redução de risco de lesão ou dores lombares e relaxamento do estresse e da tensão muscular (GÓMEZ-CONESA; MOYA, 2005).

método estático é realizado com uma movimentação lenta até o limite de desconforto e a posterior manutenção da postura (DANTAS et al., 2008). Este limite é alcançado de forma subjetiva, pois a relação entre tensão aplicada e as informações subjetivas obtidas neste método são confiáveis, seguras e possíveis de 
serem reproduzidas (BRANCO et al., 2006). A lentidão do movimento e a tensão exercida sobre o tendão fazem com que seja facilitada a ação do órgão tendinoso de Golgi (OTG), o qual produz o relaxamento do músculo que está sendo estirado, proporcionando uma maior facilidade na execução do movimento, conferindo a este uma boa eficiência (DAVIS et al., 2005).

Nesse sentido, o tempo de permanência no limite de desconforto para proporcionar ganhos na ADM ainda não está muito bem definido. Madding et al. ( 1987 ) concluíram em seu estudo que uma repetição com permanência estática de 15, 45 ou 120 segundos, produz aumentos significativos na $\operatorname{ADM}\left(7.0^{\circ}, 5.4^{\circ}\right.$ e 7, $2^{\circ}$ respectivamente). Com este resultado pode-se notar que com uma única repetição de I 5 segundos, o ganho foi tão significativo quanto 120 segundos e maior que 45 segundos. No entanto, Conceição et al. (2008) compararam o comportamento da flexibilidade utilizando o método estático com uma repetição de 10, 20, 40 e 60 segundos de permanência, três vezes por semana, e verificaram que não houve diferença significativa entre os grupos, embora todos tenham obtido aumentos na ADM. Já Taylor et al. ( 1990) observaram a adaptação do tecido conjuntivo e muscular nos primeiros estímulos realizados em cada sessão de treinamento parecendo ser em torno de quatro o número ideal de repetições por exercício com duração de 12 a 18 segundos de permanência estática. Entretanto, Roberts e Wilson (1999) estudaram três repetições de 15 segundos de permanência estática e nove de cinco, em 24 universitários e concluíram que três repetições de 15 segundos foram mais efetivos do que nove de cinco, mostrando que apesar do tempo total ter sido de 45 segundos, repetições maiores não necessariamente são mais eficientes.

Diante do exposto, nota-se que ainda não há um consenso sobre o tempo de permanência e a quantidade de repetições ideais para a otimização dos níveis de flexibilidade muscular através do método estático. Sendo assim, este estudo teve por objetivo investigar os efeitos de três repetições de 10 segundos de permanência do método estático sobre os ganhos de ADM em adultos jovens não atletas.

\section{MATERIAIS E MÉTODOS}

\section{SUJEITOS}

A amostra não probabilística foi composta por 59 homens adultos não atletas funcionários de uma grande empresa, cuja principal função era ensacar e transportar sacos de café. Os sujeitos foram divididos em dois grupos: grupo controle ( $G C ; n=18)$, o qual não foi submetido a nenhum tipo de treinamento de flexibilidade; e o grupo experimental (GE; $n=4$ I), que realizou três repetições do 
método estático com 10 segundos de permanência na posição, tendo 10 segundos de descanso entre elas, sempre efetuados na parte da manhã, três vezes por semana em dias alternados (Tabela I).

Tabela I: Características antropométricas dos grupos experimental (GE; $n=4$ I) e controle (GC; $n=\mid 8)$.

\begin{tabular}{lll}
\hline Grupo & Variáveis & Média $\pm(\mathrm{DP})$ \\
\hline GE & Massa Corporal $(\mathrm{kg})$ & $69,43 \pm 13,75$ \\
& Estatura $(\mathrm{m})$ & $1,74 \pm 0,07$ \\
$\mathrm{GC}$ & IMC & $22,88 \pm 3,96$ \\
& Massa Corporal $(\mathrm{kg})$ & $70,67 \pm 3,65$ \\
& Estatura $(\mathrm{m})$ & $1,75 \pm 12,43$ \\
& IMC & $22,96 \pm 0,08$ \\
\hline
\end{tabular}

IMC: Índice de massa corporal. DP: desvio padrão.

Foram excluídos da amostra os voluntários que, através de um breve questionário, respondiam se tinham menos de 18 e mais de 30 anos de idade, que relataram algum problema de saúde ou que praticavam alguma atividade física regular além da ginástica laboral oferecida pela empresa.

O estudo foi submetido e aprovado pelo Comitê de Ética em Pesquisa da Universidade Castelo Branco Protocolo n 0022/2007 e todos os procedimentos foram aprovados de acordo com a Resolução No 196, de 10 de outubro de 1996 (BRASIL, 1996).

\section{PROCEDIMENTOS DE COLETA DE DADOS}

Os indivíduos foram entrevistados, inicialmente, através de um questionário que continha as seguintes perguntas: I - Qual sua idade? 2- Possui alguma dor ou doença que lhe impeça de fazer atividade física? 3- Faz algum programa de exercício regularmente? 4- Seus dados podem ser utilizados em prol de nossa pesquisa? $\bigcirc$ funcionário assinava o questionário.

Após esta etapa, todos assinaram o Termo de Consentimento Livre e Esclarecido e, para fins de caracterização da amostra, tiveram seu massa corporal total aferida por uma balança digital (Filizolla, Brasil), com precisão resolução de 100 gramas. A estatura foi aferida através de um estadiômetro de parede (Cardiomed, Brasil), com precisão em centímetros. A partir destes dados foi calculado o índice de massa corporal (IMC). Todos os pontos de coleta obedeceram às recomendações da International Standards for Anthropometric Assessment (ISAK) (MARFELL-JONES et al., 2006).

O critério utilizado para divisão dos grupos foi manter os funcionários em seus galpões de trabalho, pois os funcionários exerciam as mesmas funções, mas trabalhavam em galpões diferentes, de acordo como tipo do grão de café, porém o 
esforço era o mesmo para todos os galpões. $\bigcirc$ trabalho consistia em abrir um saco de estopa de 50 kg, enchê-lo através de um cano de onde saem os grãos, fechar o saco em uma máquina própria e empurrar para uma esteira, onde o saco de café é levado até um carrinho do tipo empilhadeira para ser colocado em caminhões.

A flexibilidade foi aferida no início e no final do período de intervenção por um goniômetro de 16 polegadas, com $360^{\circ}$ de aço (Lafayette, USA) através do protocolo de goniometria LABIFIE (DANTAS; CARVALHO; FONSECA, 1997) para verificar a amplitude articular, em graus, dos seguintes movimentos: abdução do ombro (AO) - o testado estava de pé, com o braço direito ao longo do tronco, o cotovelo estendido em que o eixo central do goniômetro foi alinhado com o ponto acromial na face posterior do braço direito onde a haste fixa foi posicionada nas costas do avaliado, no sentido transversal, sobre a linha traçada entre os pontos acromiais e a haste móvel foi posicionada na parte posterior do braço sobre uma linha traçada do ponto acromial até o processo olecraniano durante todo o movimento; e flexão de quadril (FQ) - o testado permanecia deitado em decúbito dorsal, com as pernas estendidas em que o eixo central do goniômetro foi alinhado sobre o ponto trocantérico direito onde a haste fixa foi posicionada na parte lateral do tronco, sobre o prolongamento da linha axilar e a haste móvel foi posicionada na face externa da coxa em sua linha mediana durante todo o movimento.

\section{INTERVENÇÃO}

O GE realizou a intervenção por um período de 16 semanas, sempre pela manhã, antes da jornada de trabalho. Os exercícios tiveram uma frequência de três vezes na semana, em dias alternados com 48 horas de intervalo entre as sessões. A sessão de treinamento do GE foi precedida por um aquecimento de cinco minutos com exercícios suaves de mobilidade articular geral. A parte específica do treinamento de flexibilidade teve um tempo total de 120 segundos de duração por sessão e foi dividida em dois exercícios de alongamento aplicados com o método estático com três repetições de 10 segundos de tempo de permanência na posição de cada movimento, tendo 10 segundos de intervalo entre as repetições. Os exercícios são descritos a seguir.

Exercício I : feito individualmente, com o indivíduo em pé, realizava-se uma abdução de ombro com o cotovelo flexionado e o forçamento era feito pelo próprio indivíduo com a mão oposta (Figura I), permanecendo na posição durante 10 segundos e repetindo três vezes, tendo 10 segundos de intervalo entre as repetições. O exercício foi realizado em ambos os membros superiores. 


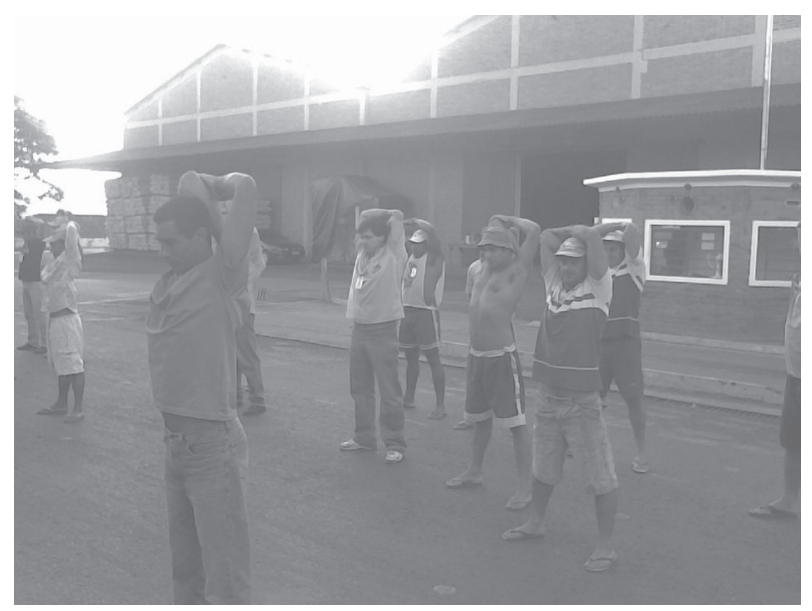

Figura I. exercício I.

Exercício 2: realizado em pé, individualmente, com uma das pernas estendidas a frente tendo o pé em dorsiflexão, enquanto que, por trás, a outra perna realizava a flexão do joelho e o tronco era flexionado à frente. Os indivíduos eram orientados a tentar encostar a testa no joelho, sem que o joelho da frente flexionasse, e levar as mãos ao pé em dorsiflexão (Figura 2). Cada movimento era mantido por 10 segundos em ambas as pernas e repetido três vezes, tendo 10 segundos de intervalo entre as repetições. $\bigcirc$ exercício foi realizado em ambos os membros inferiores.

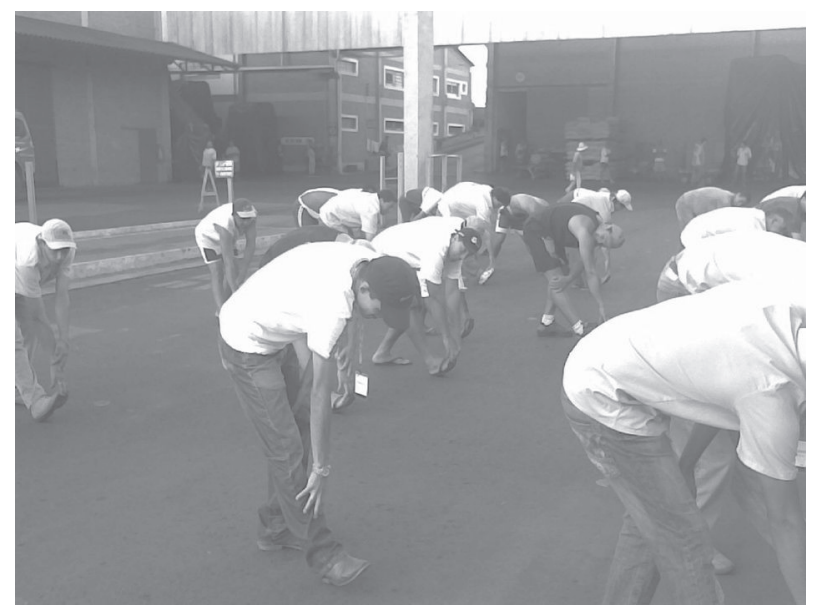

Figura 2. exercício 2. 
Todos os exercícios foram demonstrados, orientados e supervisionados por um profissional de Educação Física, que explicava que o exercício deveria ser levado até o ponto de desconforto conforme a sensação subjetiva de dor (BRANCO et al., 2006) e ser mantido por 10 segundos.

O grupo controle também era composto por ensacadores e transportadores de café e se comprometeu em manter seus afazeres diários, porém sem participar de qualquer programa de atividade física durante o período do estudo.

\section{TRATAMENTO ESTATÍSTICO}

Os dados foram tratados pelo programa PASW Statistics 18.0 for Windows e apresentados como média e desvio padrão. Utilizou-se o teste de Shapiro-Wilk e de Levene para verificar a normalidade e a homogeneidade de variância dos dados da amostra, respectivamente. Empregou-se a análise de variância com medidas repetidas nos fatores grupo (GE e GC) e tempo (pré e pós-teste), seguida do post hoc de Scheffé para identificar as possíveis diferenças. Adotou-se o valor de $\mathrm{p}<0,05$ para significância estatística.

\section{RESULTADOS}

A Tabela 2 apresenta a comparação dos níveis de flexibilidade no GE e no GC. Pode-se notar que houve aumento significativo dos níveis de flexibilidade nos movimentos $\mathrm{AO}(\Delta \%=8,9 ; \mathrm{p}=0,00)$ e FQ $(\Delta \%=18,7 ; \mathrm{p}=0,00)$ no GE. $\mathrm{O}$ GC manteve seus níveis de flexibilidade sem diferenças estatisticamente significativas $\mathrm{AO}(\Delta \%=0,4 ; p=0,68)$ e $\mathrm{FQ}(\Delta \%=-4,0 ; p=0,12)$ do pré para o pós-teste. $\mathrm{Na}$ análise intergrupo, observa-se que no pré-teste o GE apresentou valores significativamente menores que o $G C(p=0,00)$, no entanto, o mesmo não ocorreu após o período de intervenção no pós-teste.

Tabela 2. Análise comparativa dos níveis de amplitude de movimento da abdução do ombro (AO) e flexão do quadril (FQ) nos grupos experimental (GE) e controle (GC).

\begin{tabular}{llll}
\hline & $\mathrm{n}$ & Média-pré (DP) & Média-pós (DP) \\
\hline AO-GE & 41 & $205,51 \pm 20,15^{* *}$ & $223,71 \pm 13,35^{*}$ \\
AO-GC & 18 & $225,22 \pm 16,63$ & $226,11 \pm 15,29$ \\
FQ-GE & 41 & $86,59 \pm 13,55^{* *}$ & $102,80 \pm 12,69 *$ \\
FQ-GC & 18 & $97,78 \pm 11,07$ & $93,89 \pm 11,00$ \\
\hline
\end{tabular}

$\mathrm{AO}=$ abdução de ombro; $\mathrm{FQ}$ = flexão de quadril; $\mathrm{GE}=$ grupo de estudo: $\mathrm{GC}=$ grupo controle; valores em graus; DP: desvio padrão. * $p<0,05$, pré vs pós. ** $p<0,05$, GE vs $G C$. 
O estudo demonstrou que o programa de treinamento de flexibilidade de três repetições de 10 segundos de permanência estática, com 10 segundos de intervalo entre elas, através do método estático para desenvolvimento da flexibilidade em homens adultos jovens, após 16 semanas de intervenção, proporcionou um aumento significativo na ADM dos movimentos analisados. Estes achados são corroborados por Dantas e Soares (200 I ) que afirmam que o método estático deve ser repetido de três a seis vezes, com intervalo de descontração entre elas.

Roberts e Wilson (1999) compararam três repetições de 15 segundos de permanência estática com nove repetições de cinco segundos do método estático. Os autores concluíram que ambos os grupos obtiveram aumento dos níveis de flexibilidade quando comparados ao grupo controle, porém quando comparados entre eles, o grupo de três repetições obteve resultados maiores do que o grupo de nove repetições de cinco segundos. Embora não haja consenso na literatura sobre a quantidade de repetições que devam ser usadas no método estático para um rendimento ótimo de flexibilidade, nota-se que muitos estudos colocam em prática a realização de três repetições (BEST et al., 1994; BONVICINI; GONÇALVES; BATÁGLIA, 2005; SHRIER; GOSSAL, 2000). Dessa forma, a presente pesquisa que utilizou três repetições também encontrou os mesmos resultados dos estudos citados.

Observou-se que os dois movimentos analisados na presente investigação apresentaram aumentos de ADM no GE. Isto pode ter ocorrido em função das características específicas geradas através do método estático, pois exercícios de flexibilidade utilizados com intensidade baixa e tempo prolongado aumentam a deformação dos componentes plásticos do tecido não contrátil, permitindo remodelamento gradual das ligações de colágeno e redistribuição hídrica para os tecidos vizinhos (BEST et al., 1994). Nesse sentido, a extensibilidade dos tecidos moles existentes nas duas articulações como as ligações acto-miosinicas, as proteínas não contráteis do citoesqueleto (dentro e fora do sarcômero), tecidos conectivos (TAYLOR et al., 1990) podem ter influenciado o desenvolvimento dos níveis de flexibilidade no GE.

O presente estudo utilizou um tempo curto de permanência ( 10 segundos) e a intensidade foi controlada subjetivamente, pois foi solicitado aos sujeitos que levassem a articulação até o ponto de desconforto conforme recomendações de Branco et al. (2006) e encontrou aumentos de ADM no GE. Estes achados são corroborados por Conceição. (2008) que avaliaram adultos jovens através do método estático com uma repetição, mas que foram divididos em grupos conforme o tempo de permanência de 10 (G I0), 20 (G20), 40 (G40) e 60 (G60) segundos. Foram 
encontrados aumentos significativos de ADM após 16 semanas de treinamento em todos os tempos utilizados, entretanto não foram observadas diferenças significativas entre os grupos. Isso sugere que o tempo I 0 segundos de permanência parece ser suficiente para gerar aumentos de ADM.

Outro importante fator apresentado no presente estudo foi a atividade muscular desempenhada pelo tipo de trabalho profissional, que dependendo de suas características pode ser um fator que venha a influenciar a capacidade de manutenção da amplitude articular. Nesse sentido, os indivíduos da amostra do presente estudo tinham uma atividade que requeria dos mesmos ADM e solicitações musculares semelhantes, principalmente de força, desempenhadas durante a jornada de oito horas de trabalho. Dessa forma, é possível supor que a função de ensacador permite alcançar e manter níveis adequados de flexibilidade como em sujeitos submetidos a treinamentos com peso, assim como a exemplo o estudo de Cyrino et al. (2004) que observaram aumento dos níveis de flexibilidade em 16 homens com idade média de 23 anos submetidos a 10 semanas de treinamento com pesos.

\section{CONCLUSÃO}

método estático aplicado com três repetições de 10 segundos de permanência mostrou-se eficiente para o aumento dos níveis de flexibilidade na $\mathrm{AO}$ e FQ de homens adultos não atletas. Estes achados são úteis aos profissionais de Educação Física, que poderão aplicar o treino para aumento da flexibilidade com mais eficiência, lembrando que atividades de força e flexibilidade podem ser complementares para o desempenho funcional de trabalhadores. As atividades cotidianas devem ser observadas com atenção para que os programas de exercícios proporcionem bons resultados. Sugere-se que sejam realizados estudos comparativos entre diferentes repetições e tempos de permanência estática, assim como em outros grupos de trabalhadores.

Effect of three repetitions of ten seconds stimulus of the static method for the development of the flexibility in young adult men

ABSTRACT: The aim of the study was to investigate the effects of three repetitions of 10 seconds of remaining of the static method on range of motion gains in young adult nonathletes. Fifty nine men were divided into an experimental group ( $E G, n=4 I$ ), which was submitted to static stretching exercises for 16 weeks with application of three repetitions of 10 seconds of remaining in the position of each movement and a control group (CG, $n$ $=18$ ), that was not submitted to any training. We evaluated the movements of shoulder abduction (SA) and hip flexion (HF) through goniometry. The repeated measures ANOVA showed significant increases in EG of the pre to pos-test in $S A(\%=8,9 ; p=0,00)$ and 
HF $(\%=18,7 ; p=0,00)$. In the pos-test there were no differences between groups. We concluded that the proposed method was enough to produce significant increase in levels of flexibility in the movements analyzed.

KEYWORDS: Flexibility; stretching; range of motion.

\section{Efectos de tres repeticiones de diez segundos de estímulo del método estático para el desarrollo de la flexibilidad de hombres adultos jóvenes}

RESUMEN: Este estudio tuvo como objetivo investigar los efectos de tres repeticiones de 10 segundos de duración del método estático sobre las ganancias en el rango de movimiento en los adultos jóvenes no atletas. Los 59 hombres fueron divididos en un grupo de estudio (GE) ( $n=4$ I) que fue sometido a la 16 semanas de ejercicios de estiramiento del método estático, con tres repeticiones y duración de diez segundos de permanencia en la posición de cada movimiento y un grupo control (GC) $(n=18)$ que no fue sometido a ningún entrenamiento. Fueran evaluados los movimientos de abducción del hombro (AH) y flexión de cadera (FC) a través de goniometría. La ANOVA de medidas repetidas reveló un aumento significativo en el GE del pre para el pos-test en la $\mathrm{AH}(\%=8.9, p=0.00)$ y $F C(=18,7 \%, p=0.00)$. En el pos-test no se observaron diferencias entre los grupos. Se concluye que el método propuesto fue suficiente para producir aumento significativo en los niveles de flexibilidad en los movimientos analizados.

PALABRAS CLAVE: Flexibilidad; estiramiento; rango de movimiento.

\section{REFERÊNCIAS}

BEST, T. M. J. et al. Characterization of passive responses of live skeletal muscle using the quasi-linear theory of viscoelasticity. Journal of Biomechanics. Amsterdam, v. 27, n. 4, p. 413-419, 1994.

BONVICINI, C.; GONÇALVES, C.; BATÁGLIA F. Comparação do ganho de flexibilidade isquiotibial com diferentes técnicas de alongamento passivo. Acta Fisiátrica. São Paulo, v. I2, n. 2, p. 43-47, 2005.

BRANCO, V. R. et al. Relação entre a tensão aplicada e a sensação de desconforto nos músculos isquiotibiais durante o alongamento. Revista Brasileira de Fisioterapia. São Carlos, v. 10, n. 4, p. 65-472, 2006.

CONCEIÇÃO, M. C. S. C. et al. Efeitos de quatro tempos diferentes de permanência de flexionamento estático na flexibilidade de adultos jovens. Fitness and Performance Journal. Rio de Janeiro, v. 7, n. 2, p. 88-92, 2008.

CYRINO, E.S. et al. Comportamento da flexibilidade após 10 semanas de treinamento com pesos. Revista Brasileira de Medicina do Esporte. Niterói, v. I0, n. 4, p. 87- 99, 2004. 
DANTAS, E. H. M.; CARVALHO, J. T. L.; FONSECA, R. M. O protocolo LABIFIE de goniometria. Revista Treinamento Desportivo. Curitiba, v. 2, n. 3, p. 21-34, 1997.

DANTAS, E. H. M.; SOARES, J.S. Flexibilidade aplicada ao personal trainer. Fitness and Performance Journal. Rio de Janeiro, v. 0, n. I, p. 7-12, 2001.

DANTAS, E. H. M. et al. Scale of perceived exertion in the flexibility (PERFLEX): a dimensionless tool to evaluate the intensity? Fitness and Performance Journal. Rio de Janeiro, v. 7, n. 5 , p. 289-94, 2008. doi: 10.3900/fpj.7.5.289.e.

DAVIS, D. S. et al. The effectiveness of 3 stretching techniques on hamstring flexibility using consistent stretching parameters. Journal of Strength and Conditioning Research. Philadelphia, v. 19, n. I, p. 27-32, 2005.

GÓMEZ-CONESA, A.; MOYA, S.V. Lumbalgia crônica y discapacidad laboral. Fisioterapia. Madrid, v. 27, n. 5, p. 255-65, 2005.

GONÇALVES, R.; GURJÃO, A. L. D.; GOBBI, S. Efeitos de oito semanas do treinamento de força na flexibilidade de idosos. Revista Brasileira de Cineantropometria e Desempenho Humano, Florianópolis, v. 9, n. 2, p. 145-153, 2007.

GUTIÉRREZ NIETO, M. et al. Propuesta de clasificación de las técnicas de estiramiento en fisioterapia. Fisioterapia, Madrid, v. 25, n. 4, p. 199-208, 2003.

HAFF, G. Roundtable discussion: flexibility training. Strength and Conditioning Journal. Philadelphia, v. 28, p. 64-85, 2006.

LIMA, R. C. M. et al. Análise da durabilidade do efeito do alongamento muscular dos isquiotibiais em duas formas de intervenção. Acta Fisiatrica. São Paulo, v. I3, n. I, p. 32-38, 2006.

MADDING, S. W. et al. Effect of duration of passive stretch on hip abduction range of motion. Journal of Orthopaedic and Sports Physical Therapy. Alexandria, v. 8, p. 409-4I6, 1987.

MARFELL-JONES, M. et al. International standards for anthropometric assessment. Potchefstroom. South África: ISAK; 2006.

OSTERNING, L. R.; ROBERTSON, R. N.; TROXEL, R. K. Differential responses to proprioceptive neuromuscular facilitation (PNF) stretches techniques. Medicine and Science in Sports and Exercise. Madison, v. 22, p. 106-1।1, 1990.

ROBERTS, J, M,; WILSON, K. Effect of stretching duration on active and passive range of motion in the lower extremity. British Journal of Sports Medicine. London, v. 33, p. 259$263,1999$.

SHRIER, I.; GOSSAL, K. Myths and truths of stretching: individualized recommendations for healthy muscles. Physician and Sportsmedicine. New York, v. 28, n. 8, p. 35-46, 2000. 
TAYLOR, D. C. et al. Viscoelastic properties of muscle tendon units. The biomechanical effects of stretching. The American Journal of Sports Medicine. London, v. I8, n. 3, p. 300-308, 1990.

VIVEIROS, L. et al. Respostas agudas imediatas e tardias da flexibilidade na extensão do ombro em relação ao número de séries e duração do alongamento. Revista Brasileira de Medicina do Esporte. Niterói, v. I0, n. 6, p. 459-463, 2004.

Recebido: II abr. 2010 Aprovado: 24 ago. 2010

Endereço para correspondência:

Rodrigo Gomes de Souza Vale Rua Oscar Clark, 805 - Parque Mataruna Araruama-RJ CEP: 28970-000 